\title{
Outage probability analysis of DF PSR energy harvesting Full-Duplex relaying network with presence of the direct link using MRC technique
}

\author{
Van-Duc Phan ${ }^{1}$, Phu Tran Tin ${ }^{2}$, Minh Tran ${ }^{3}$, and Tran Thanh Trang ${ }^{4}$ \\ ${ }^{1}$ Center of Excellence for Automation and Precision Mechanical Engineering, \\ Nguyen Tat Thanh University, Vietnam \\ ${ }^{2}$ Wireless Communications Research Group, Faculty of Electrical and Electronics Engineering, \\ Ton Duc Thang University, Vietnam \\ ${ }^{3}$ Optoelectronics Research Group, Faculty of Electrical and Electronics Engineering, \\ Ton Duc Thang University, Vietnam \\ ${ }^{4}$ National Key Laboratory of Digital Control and System Engineering, Vietnam
}

\begin{tabular}{l} 
Article Info \\
\hline Article history: \\
Received Jan 26, 2019 \\
Revised Apr 22, 2019 \\
Accepted May 7, 2019 \\
\hline Keywords: \\
Decode-and-forward (DF) \\
Energy harvesting (EH) \\
Outage probability (OP) \\
Power splitting protocol (PS) \\
Relaying network
\end{tabular}

Article Info

Article history:

Received Jan 26, 2019

Revised Apr 22, 2019

Accepted May 7, 2019

\section{Relaying network}

\begin{abstract}
In the last time, the system performance of the energy harvesting relay network has been considered in many studies. In this paper, we propose and investigate the outage probability (OP) of the Decode-and-Forward (DF) Energy Harvesting (EH) Full-Duplex (FD) Relaying network in Power Splitting Protocol (PS) using MRC Technique with the presence of the direct link. In the first stage, the integral form of the OP is derived in two cases with and without the presence of the direct link. After that, we analyze the influence of main system parameters on the OP and comparison between two cases with and without the presence of the direct link. Finally, the results show that all simulation and analytical results match well with each other based on the Monte Carlo verification simulation.
\end{abstract}

Copyright $@ 2019$ Institute of Advanced Engineering and Science. All rights reserved.

\section{Corresponding Author:}

Phu Tran Tin,

Wireless Communications Research Group,

Faculty of Electrical and Electronics Engineering,

Ton Duc Thang University, Ho Chi Minh City, Vietnam.

Email: phutrantin@tdtu.edu.vn

\section{INTRODUCTION}

In the last decades, the Internet of Things (IoT) is a too hot research area over the world. With its potential to significantly influence all aspects of our daily lives, IoT is expected to have a significant impact on businesses by automating some processes and improving the control of many environment variables [1-6]. The wireless powered communication network (WPCN), where network devices harvest energy from the signals transmitted by RF energy sources in the first step and then utilize this harvested energy for their communication needs, is the primary direction in the RF energy harvesting. In the last decade, many research papers focused on WPCN and how to improve its efficiency. This concept of a tradeoff between EH and information transmission in WPCN was proposed and investigated in [7] and extended in [8]. Moreover, the concept of partial network level cooperation for EH networks was presented in detail in [9], and in [10] wireless $\mathrm{EH}$ and information transfer in cognitive relay networks was intensely analyzed. In WPCN, the two traditional time switching (TSP) and power splitting (PSP) protocols have been intensively studied in the literature, and many from these studies have compared the system performance of the two protocols under different scenarios [11-15]. 
In this work, we propose and investigate the outage probability (OP) of the Decode-and-Forward (DF) Energy Harvesting (EH) Full-Duplex (FD) Relaying network in Power Splitting Protocol (PS) using MRC Technique with the presence of the direct link. In the first stage, the integral form of the OP is derived in two cases with and without the presence of the direct link. After that, we analyze the influence of main system parameters on the OP and comparison between two cases with and without the presence of the direct link. Finally, the results show that all simulation and analytical results match well with each other based on the Monte Carlo verification simulation. The structure of this paper is proposed as follows. Sections II presents the system model of the relaying network. Sections III proposes the analytical expression of OP in cases with and without the direct link between S and D. Section IV provides the numerical results and some discussions. Finally, Section V concludes the paper.

\section{SYSTEM MODEL}

Figure 1 plots the DF EH FD relaying network in the PS Protocol using MRC technique with the presence of the direct link. In this model system, the information is transferred from the source (S) to the destination (D), through energy constrained intermediate relay (R) and with the direct link. The energy harvesting and information processing of the system model with PS protocol are illustrated in Figure 2. In this scheme, $\mathrm{T}$ is the block time in which the source fully transmit the information data to the destination. In the first interval time $(T / 2)$, the relay harvests energy $(\rho T)$ and transfers the information $((1-\rho) \mathrm{T})$ from the source signal to the destination, where $\rho$ is the power splitting factor $\rho \in(0,1)$. Here, we consider the interference noise at the relay node. In the remaining half-time $\mathrm{T} / 2$, the direct link transfers information from the source to the destination node. All the fading channels from $\mathrm{S}$ to $\mathrm{R}$ and $\mathrm{R}$ to $\mathrm{D}$ are proposed as the Raleigh fading channels [16-20].

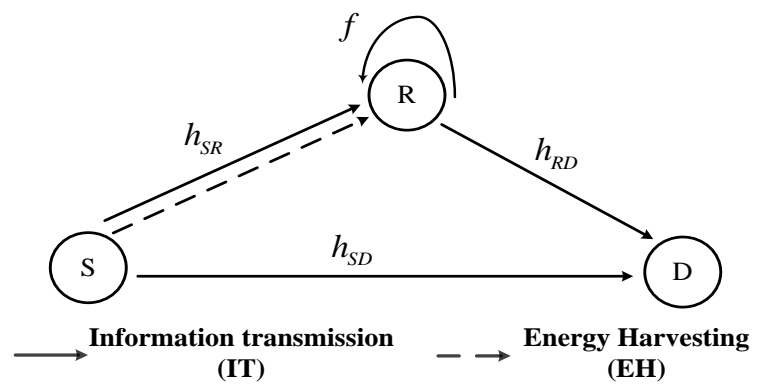

Figure 1. System model

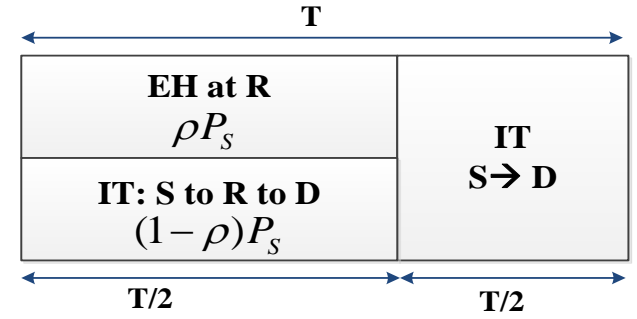

Figure 2. The power splitting protocol

\section{THE SYSTEM PERFORMANCE}

\subsection{Energy Harvesting Phase}

In the first time slot, the received signal at the relay can be expressed as

$$
y_{r}=\sqrt{\rho P_{s}} h_{S R} x_{s}+n_{r}
$$


Where $x_{s}$ is the transmit signal at the source and where $\mathrm{E}\left\{\left|x_{s}\right|^{2}\right\}=1, \mathrm{E}\{\bullet\}$ is the expectation operator, Ps is transmitted power of the source, ${ }_{r}$ is the additive white Gaussian noise (AWGN) at the relay $\mathrm{R}$ with zero-mean and variance N0.

The harvested energy at the relay can be given by

$$
E_{r}=\eta \rho(T / 2) P_{s}\left|h_{S R}\right|^{2}
$$

From (2), the average transmitted power at the relay can be obtained as

$$
P_{r}=\frac{E_{r}}{T / 2}=\eta \rho P_{s}\left|h_{S R}\right|^{2}
$$

Where $0<\eta \leq 1$ is the energy conversion efficiency and $0<\rho<1$ is the power splitting factor.

\subsection{Information Transmission Phase}

During the information transmission phase in the first time slot, the received signal at the relay can be given as [21-27]

$$
y_{r}=\sqrt{(1-\rho) P_{s}} h_{S R} x_{s}+f \sqrt{P_{r}} x_{r}+n_{r}
$$

Where $x_{r}$ is the transmit signal at the source and $\mathrm{E}\left\{\left|x_{r}\right|^{2}\right\}=1$, $\mathrm{f}$ is loopback interference channel. In the first time slot, the received signal at the destination in whole this time can be given as

$$
y_{d}^{1}=\sqrt{P_{r}} h_{R D} x_{r}+n_{d}^{1}
$$

Where $n_{d}^{1}$ is the additive white Gaussian noise (AWGN) at the destination in the first time slot with zero-mean and variance NO.

In the second time slot, the destination will be received the signal directly from the source, which can be calculated as

$$
y_{d}^{2}=\sqrt{P_{s}} h_{S D} x_{s}+n_{d}^{2}
$$

Where $n_{d}^{2}$ is the AWGN at the destination in the second time slot with zero-mean and variance N0.

From (4), the signal to noise ratio (SNR) at the relay can be computed as the following

$$
\gamma_{S R}=\frac{(1-\rho) P_{s}\left|h_{S R}\right|^{2}}{P_{r}|f|^{2}+N_{0}}
$$

Substituting (3) into (7) and using the fact that N0<<Ps, (7) can be rewritten as

$$
\gamma_{S R} \approx \frac{(1-\rho)}{\eta \rho|f|^{2}}
$$

From (5), the SNR at the destination in the first time slot can be calculated as the below equation:

$$
\gamma_{R D}=\frac{P_{r}\left|h_{R D}\right|^{2}}{N_{0}}=\frac{\eta \rho P_{s}\left|h_{S R}\right|^{2}\left|h_{R D}\right|^{2}}{N_{0}}=\psi \eta \rho\left|h_{S R}\right|^{2}\left|h_{R D}\right|^{2}
$$


Where we denote

$$
\psi=\frac{P_{s}}{N_{0}} .
$$

From (6), the SNR at the destination in the second time slot can be claimed as

$$
\gamma_{S D}=\frac{P_{s}\left|h_{S D}\right|^{2}}{N_{0}}=\psi\left|h_{S D}\right|^{2}
$$

Using the MRC technique at the destination, the end to end SNR can be expressed as

$$
\gamma_{e 2 e}= \begin{cases}\gamma_{R D}+\gamma_{S D}, & \text { if } \gamma_{S R} \geq \gamma_{0} \\ \gamma_{S D}, & \text { if } \gamma_{S R}<\gamma_{0}\end{cases}
$$

Where $\gamma_{0}=2^{2 R}-1$ is the threshold of system and $\mathrm{R}$ is the target rate.

\subsection{Outage Probability Analysis}

$$
\begin{aligned}
O P & =\operatorname{Pr}\left(\gamma_{S D}<\gamma_{0}, \gamma_{S R}<\gamma_{0}\right)+\operatorname{Pr}\left(\gamma_{S D}+\gamma_{R D}<\gamma_{0}, \gamma_{S R} \geq \gamma_{0}\right) \\
& =P_{1}+P_{2}
\end{aligned}
$$

Where $P_{1}=\operatorname{Pr}\left(\gamma_{S D}<\gamma_{0}, \gamma_{S R}<\gamma_{0}\right)$ and $P_{2}=\operatorname{Pr}\left(\gamma_{S D}+\gamma_{R D}<\gamma_{0}, \gamma_{S R} \geq \gamma_{0}\right)$.

Substituting (8) and (10) into P1, we have

$$
\begin{aligned}
P_{1} & =\operatorname{Pr}\left[\frac{(1-\rho)}{\eta \rho|f|^{2}}<\gamma_{0}, \psi\left|h_{S D}\right|^{2}<\gamma_{0}\right]=\operatorname{Pr}\left[\frac{(1-\rho)}{\eta \rho|f|^{2}}<\gamma_{0}\right] \times \operatorname{Pr}\left(\psi\left|h_{S D}\right|^{2}<\gamma_{0}\right) \\
& =\left\{1-\operatorname{Pr}\left[|f|^{2} \leq \frac{(1-\rho)}{\eta \rho \gamma_{0}}\right]\right\} \times \operatorname{Pr}\left(\left|h_{S D}\right|^{2}<\frac{\gamma_{0}}{\psi}\right) \\
& =\left[1-F_{|f|^{2}}\left(\frac{(1-\rho)}{\eta \rho \gamma_{0}}\right)\right] \times F_{\left|h_{S D}\right|^{2}}\left(\frac{\gamma_{0}}{\psi}\right)=\exp \left[\frac{\lambda_{f}(\rho-1)}{\eta \rho \gamma_{0}}\right] \times\left[1-\exp \left(-\frac{\lambda_{S D} \gamma_{0}}{\psi}\right)\right]
\end{aligned}
$$

Where $\lambda_{f}, \lambda_{S D}$ are the mean of the random variable (RV) $|f|^{2}$ and $\left|h_{S D}\right|^{2}$, respectively. Substituting (8), (9) and (10) into P2, we can obtain P2 as followings

$$
\begin{aligned}
P_{2} & =\operatorname{Pr}\left(\psi\left|h_{S D}\right|^{2}+\psi \eta \rho\left|h_{S R}\right|^{2}\left|h_{R D}\right|^{2}<\gamma_{0}, \frac{(1-\rho)}{\eta \rho|f|^{2}} \geq \gamma_{0}\right) \\
& =\operatorname{Pr}\left(\psi\left|h_{S D}\right|^{2}+\psi \eta \rho\left|h_{S R}\right|^{2}\left|h_{R D}\right|^{2}<\gamma_{0}\right) \times \operatorname{Pr}\left[\frac{(1-\rho)}{\eta \rho|f|^{2}} \geq \gamma_{0}\right] \\
& =\left[1-\exp \left(\frac{\lambda_{f}(\rho-1)}{\eta \rho \gamma_{0}}\right)\right] \times \operatorname{Pr}\left(\psi\left|h_{S D}\right|^{2}+\psi \eta \rho\left|h_{S R}\right|^{2}\left|h_{R D}\right|^{2}<\gamma_{0}\right)
\end{aligned}
$$

Here, we consider

$$
\begin{aligned}
P_{3} & =\operatorname{Pr}\left(\psi\left|h_{S D}\right|^{2}+\psi \eta \rho\left|h_{S R}\right|^{2}\left|h_{R D}\right|^{2}<\gamma_{0}\right)=\operatorname{Pr}\left(\psi \eta \rho\left|h_{S R}\right|^{2}\left|h_{R D}\right|^{2}<\gamma_{0}-\psi\left|h_{S D}\right|^{2}\right) \\
& =\int_{0}^{\gamma_{0}} f_{X}(x) d x \int_{0}^{\gamma_{0}-x} f_{Y}(y) d y=\int_{0}^{\gamma_{0}}\left[F_{Y}\left(\gamma_{0}-x\right)-F_{Y}(0)\right] f_{X}(x) d x
\end{aligned}
$$


Where we denote $X=\psi \eta \rho\left|h_{S R}\right|^{2}\left|h_{R D}\right|^{2}, Y=\psi\left|h_{S D}\right|^{2}$ and

$F_{Y}(y)=\operatorname{Pr}(Y<y)=\operatorname{Pr}\left(\psi\left|h_{S D}\right|^{2}<y\right)=1-\exp \left(-\frac{\lambda_{S D} y}{\psi}\right)$

Easily to observe that $F_{Y}(0)=0$. Hence, (15) can be rewritten as the following

$P_{3}=\int_{0}^{\gamma_{0}} F_{Y}\left(\gamma_{0}-x\right) f_{X}(x) d x$

Continuing, we have to find $f_{X}(x)$ as the following steps

$$
\begin{aligned}
f_{X}(x) & =\frac{\partial F_{X}(x)}{\partial x} \\
F_{X}(x) & =\operatorname{Pr}(X<x)=\operatorname{Pr}\left(\psi \eta \rho\left|h_{S R}\right|^{2}\left|h_{R D}\right|^{2}<x\right)=\operatorname{Pr}\left(\left|h_{S R}\right|^{2}<\frac{x}{\psi \eta \rho\left|h_{R D}\right|^{2}}\right) \\
& =\int_{0}^{\infty} F_{\left|h_{S R}\right|^{2}}\left[\frac{x}{\psi \eta \rho\left|h_{R D}\right|^{2}}\right] f_{\left|h_{R D}\right|^{2}}\left(\left|h_{R D}\right|^{2}\right) d\left(\left|h_{R D}\right|^{2}\right) \\
& =1-\lambda_{R D} \int_{0}^{\infty} \exp \left(-\frac{\lambda_{S R} x}{\psi \eta \rho\left|h_{R D}\right|^{2}}\right) \times \exp \left(-\lambda_{R D}\left|h_{R D}\right|^{2}\right) d\left(\left|h_{R D}\right|^{2}\right)
\end{aligned}
$$

Where $\lambda_{S R}, \lambda_{R D}$ are the mean of the random variable (RV) $\left|h_{S R}\right|^{2}$ and $\left|h_{R D}\right|^{2}$, respectively. Apply $(3.324,1)$ of the table of integrals in [28], (18) can be rewritten as the following

$$
F_{X}(x)=1-2 \sqrt{\frac{\lambda_{S R} \lambda_{R D} x}{\psi \eta \rho}} \times K_{1}\left(2 \sqrt{\frac{\lambda_{S R} \lambda_{R D} x}{\psi \eta \rho}}\right)
$$

Where $K_{v}(\bullet)$ is the modified Bessel function of the second kind and vth order.

Substituting (19) into (17) and using the formulas $\frac{d}{d x}\left(x^{v} K_{v}(x)\right)=-x^{v} K_{v-1}(x)$, we have

$$
f_{X}(x)=\frac{2 \lambda_{S R} \lambda_{R D}}{\psi \eta \rho} \times K_{0}\left(2 \sqrt{\frac{\lambda_{S R} \lambda_{R D} x}{\psi \eta \rho}}\right)
$$

Substituting (20) into (16), we can claim P3 as following

$$
P_{3}=\frac{2 \lambda_{S R} \lambda_{R D}}{\psi \eta \rho} \int_{0}^{\gamma_{0}}\left[1-\exp \left\{\frac{\lambda_{S D}\left(x-\gamma_{0}\right)}{\psi}\right\}\right] \times K_{0}\left(2 \sqrt{\frac{\lambda_{S R} \lambda_{R D} x}{\psi \eta \rho}}\right) d x
$$

Finally, substituting (21) into (14), we have

$$
P_{2}=\frac{2 \lambda_{S R} \lambda_{R D}}{\psi \eta \rho}\left[1-\exp \left(\frac{\lambda_{f}(\rho-1)}{\eta \rho \gamma_{0}}\right)\right] \times\left\langle\int_{0}^{\gamma_{0}}\left[1-\exp \left\{\frac{\lambda_{S D}\left(x-\gamma_{0}\right)}{\psi}\right\}\right] \times K_{0}\left(2 \sqrt{\frac{\lambda_{S R} \lambda_{R D} x}{\psi \eta \rho}}\right) d x\right\rangle
$$


From (13) and (22), the OP can be obtained as the following

$$
\begin{aligned}
O P & =\exp \left[\frac{\lambda_{f}(\rho-1)}{\eta \rho \gamma_{0}}\right] \times\left[1-\exp \left(-\frac{\lambda_{S D} \gamma_{0}}{\psi}\right)\right]+\frac{2 \lambda_{S R} \lambda_{R D}}{\psi \eta \rho}\left[1-\exp \left(\frac{\lambda_{f}(\rho-1)}{\eta \rho \gamma_{0}}\right)\right] \\
& \times\left\langle\int_{0}^{\gamma_{0}}\left[1-\exp \left\{\frac{\lambda_{S D}\left(x-\gamma_{0}\right)}{\psi}\right\}\right] \times K_{0}\left(2 \sqrt{\frac{\lambda_{S R} \lambda_{R D} x}{\psi \eta \rho}}\right) d x\right\rangle
\end{aligned}
$$

\section{NUMERICAL RESULTS AND DISCUSSION}

In this section, we investigate the influence of energy coefficient $\eta$ on the OP of the proposed system is plotted in Figure 1. In this paper, we set the main parameter as $\psi=5 d B, \rho=0.5, R=0.5$ and 1 . From the results, we can see that the OP has a slight decrease while $\eta$ increases from 0 to 1 . Besides, the OP of the proposed system in the case with the direct link between the $\mathrm{S}$ and $\mathrm{D}$ is better than in the case without the direct link. Moreover, the OP versus $\psi$ is illustrated in Figure 4 with the main parameters as $\eta=0.8, \rho=0.25$ and 0.85 . The results show that the OP decreases with the rising of $\psi$. However, the OP in the case with the direct link between the $\mathrm{S}$ and $\mathrm{D}$ has a considerable decrease in comparison with the OP in the case without the direct link. In all above Figures., all the simulation and analytical results are the same with both cases with and without a direct link between $\mathrm{S}$ and $\mathrm{D}$.

Furthermore, the effect of R and $\rho$ on the OP of the proposed system are drawn in Figures 5 and 6. Wee set some main parameters of the system as $\psi=5 \mathrm{~dB}, \rho=0.5, \eta=0.8$. Figure 5 shows that the Op increases crucially when $\mathrm{R}$ rises from 1 to 2 then has the same value as $\mathrm{R}$ from 2 to 7 . The OP has a slight decrease the increases when $\rho$ varies from 0 to 1 . From the above Figures., we can see that the OP in the case without direct link is better than in the case with the direct link. All the simulation result agree well with the analytical results as shown in Figures 5 and 6. OP versus $\eta$ as shown in Figure 3.

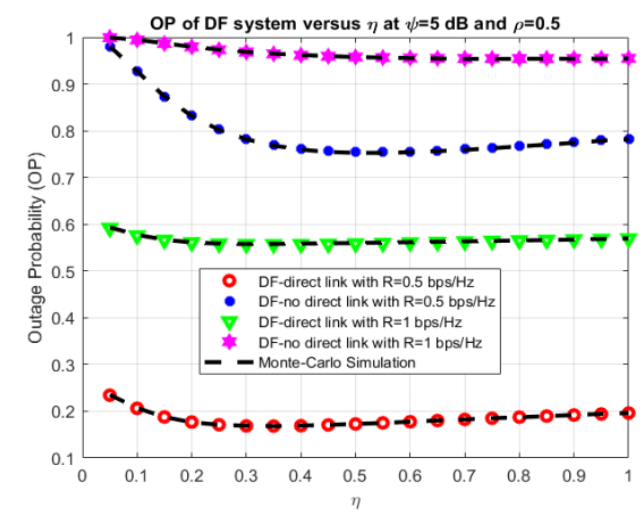

Figure 3. OP versus $\eta$

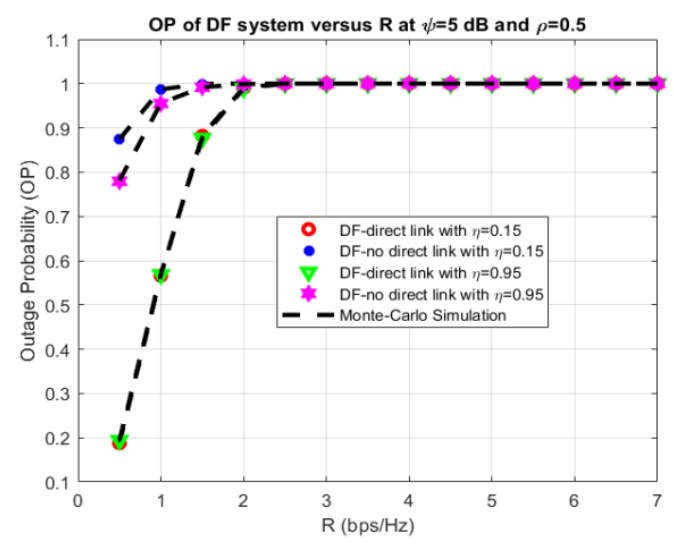

Figure 5. OP versus R

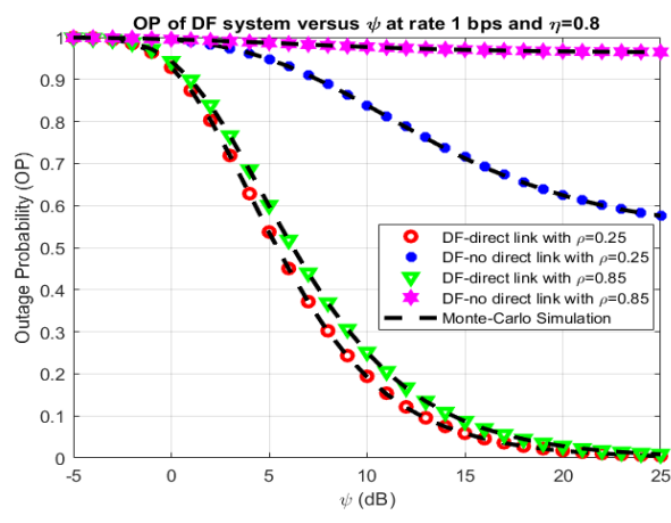

Figure 4. OP versus $\psi$

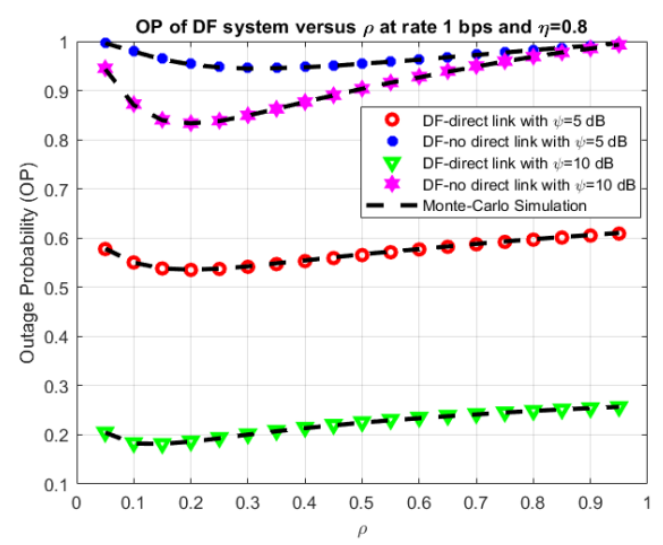

Figure 6. OP versus $\rho$ 


\section{CONCLUSION}

In this paper, we propose and investigate the OP of the DF EH FD relaying network in the PS Protocol using MRC technique with the presence of the direct link. In the first stage, the integral form of the OP is derived in two cases with and without the presence of the direct link. After that, we analyze the influence of main system parameters on the OP and comparison between two cases with and without the presence of the direct link. Finally, the results show that all simulation and analytical results match well with each other based on the Monte Carlo verification simulation. The research results can be proposed as a recommendation for manufacturing EH relaying communication network.

\section{ACKNOWLEDGEMENTS}

This research was supported by National Key Laboratory of Digital Control and System Engineering (DCSELAB), HCMUT, VNU-HCM.

\section{REFERENCES}

[1] Chen, Xiaoming, Derrick Wing Kwan Ng, and Hsiao-Hwa Chen. "Secrecy Wireless Information and Power Transfer: Challenges and Opportunities." IEEE Wireless Communications 23, no. 2 (04 2016): 54-61. doi:10.1109/mwc.2016.7462485

[2] Bi, S., Ho, C. K., \& Zhang, R. (2015, 04). "Wireless powered communication: Opportunities and challenges". IEEE Communications Magazine, 53(4), 117-125. doi:10.1109/mcom.2015.7081084.

[3] Niyato, D., Kim, D. I., Maso, M., \& Han, Z. (2017). "Wireless Powered Communication Networks: Research Directions and Technological Approaches". IEEE Wireless Communications, 2-11. doi:10.1109/mwc.2017.1600116.

[4] Atallah, R., Khabbaz, M., \& Assi, C. (2016, 04). "Energy harvesting in vehicular networks: A contemporary survey". IEEE Wireless Communications, 23(2), 70-77. doi:10.1109/mwc.2016.7462487.

[5] Tutuncuoglu, K., \& Yener, A. (2013, 09). "Cooperative energy harvesting communications with relaying and energy sharing". 2013 IEEE Information Theory Workshop (ITW). doi:10.1109/itw.2013.6691280.

[6] Nasir, Ali A., Xiangyun Zhou, Salman Durrani, and Rodney A. Kennedy. "Relaying Protocols for Wireless Energy Harvesting and Information Processing." IEEE Transactions on Wireless Communications 12, no. 7 (07 2013): 3622-636. doi:10.1109/twc.2013.062413.122042.

[7] Varshney, Lav R. "Transporting Information and Energy Simultaneously." 2008 IEEE International Symposium on Information Theory, 07 2008. doi:10.1109/isit.2008.4595260.

[8] Zhou, Xun, Rui Zhang, and Chin Keong Ho. "Wireless Information and Power Transfer: Architecture Design and Rate-energy Tradeoff." 2012 IEEE Global Communications Conference (GLOBECOM), 122012. doi:10.1109/glocom.2012.6503739.

[9] Kashef, Mohamed, and Anthony Ephremides. "Optimal Partial Relaying for Energy-Harvesting Wireless Networks." IEEE/ACM Transactions on Networking 24, no. 1 (02 2016): 113-22. doi:10.1109/tnet.2014.2361683.

[10] Wang, Zihao, Zhiyong Chen, Yao Yao, Bin Xia, and Hui Liu. "Wireless Energy Harvesting and Information Transfer in Cognitive Two-way Relay Networks." 2014 IEEE Global Communications Conference, 122014. doi:10.1109/glocom.2014.7037344.

[11] Ju, Minchul, Kyu-Min Kang, Kyu-Sung Hwang, and Cheol Jeong. "Maximum Transmission Rate of PSR/TSR Protocols in Wireless Energy Harvesting DF-Based Relay Networks." IEEE Journal on Selected Areas in Communications 33, no. 12 (12 2015): 2701-717. doi:10.1109/jsac.2015.2481213.

[12] Nguyen T.N., Do D.T., Tran P.T., and Voznak M. "Time Switching for Wireless Communications with FullDuplex Relaying in Imperfect CSI Condition." KSII Transactions on Internet and Information Systems 10, no. 9 (09, 2016). doi:10.3837/tiis.2016.09.011.

[13] Gu, Yanju, and Sonia Aissa. "RF-Based Energy Harvesting in Decode-and-Forward Relaying Systems: Ergodic and Outage Capacities." IEEE Transactions on Wireless Communications 14, no. 11 (11 2015): 6425-434. doi:10.1109/twc.2015.2453418.

[14] Zhou, Zheng, Mugen Peng, Zhongyuan Zhao, and Yong Li. "Joint Power Splitting and Antenna Selection in Energy Harvesting Relay Channels." IEEE Signal Processing Letters 22, no. 7 (07 2015): 823-27. doi:10.1109/1sp.2014.2369748.

[15] Liu, L., Zhang, R., \& Chua, K. (2013, 09). "Wireless Information and Power Transfer: A Dynamic Power Splitting Approach". IEEE Transactions on Communications, 61(9), 3990-4001. doi:10.1109/tcomm.2013.071813.130105

[16] E. Biglieri, J. Proakis, and S. Shamai, "Fading channels: information-theoretic and communications aspects," IEEE Transactions on Information Theory, vol. 44, no. 6, pp. 2619-2692, 1998.

[17] El-Malek, Ahmed H. Abd, Fawaz S. Al-Qahtani, Trung Q. Duong, Salam A. Zummo, and Hussein Alnuweiri. "MIMO Cognitive Relay Networks With Correlated Antennas Over Rayleigh Fading Channels." IEEE Transactions on Vehicular Technology 65, no. 7 (07 2016): 5349-363. doi:10.1109/tvt.2015.2450363.

[18] Duy, Tran Trung, Vo Nguyen Son, Vu Thanh Tung, George C. Alexandropoulos, and Trung Q. Duong. "Outage Performance of Cognitive Cooperative Networks with Relay Selection over Double-Rayleigh Fading Channels." IET Communications 10, no. 1 (01, 2016): 57-64. doi:10.1049/iet-com.2015.0236. 
[19] Tao, Ran, et al. "Adaptive Relaying Protocol for Wireless Power Transfer and Information Processing." IEEE Communications Letters, vol. 20, no. 10, 2016, pp. 2027-2030., doi:10.1109/lcomm.2016.2593877.

[20] Li, Tao, Pingyi Fan, and Khaled Ben Letaief. "Outage Probability of Energy Harvesting Relay-Aided Cooperative Networks Over Rayleigh Fading Channel." IEEE Transactions on Vehicular Technology 65, no. 2 (02 2016): 972-78. doi:10.1109/tvt.2015.2402274.

[21] Tan N. Nguyen, T.H.Q.Minh, Phuong T. Tran and Miroslav Voznak. "Adaptive Energy Harvesting Relaying Protocol for Two-Way Half Duplex System Network over Rician Fading Channel." Wireless Communications and Mobile Computing, 2018.

[22] Tin, Phu Tran, Tran Hoang Quang Minh, Tan N. Nguyen, and Miroslav Voznak. "System Performance Analysis of Half-Duplex Relay Network over Rician Fading Channel." TELKOMNIKA (Telecommunication, Computing, Electronics and Control), 16, no. 1 (02, 2018): 189. doi:10.12928/telkomnika.v16i1.7491.

[23] Rashid, Tarique, Sunil Kumar, Akshay Verma, Prateek Raj Gautam, and Arvind Kumar. "Pm-EEMRP: Postural Movement Based Energy Efficient Multi-hop Routing Protocol for Intra Wireless Body Sensor Network (IntraWBSN)." TELKOMNIKA (Telecommunication, Computing, Electronics and Control) 16, no. 1 (02, 2018): 166. doi:10.12928/telkomnika.v16i1.7318.

[24] F. Morabito, "Power Synthesis of Mask-Constrained Shaped Beams Through Maximally-Sparse Planar Arrays," TELKOMNIKA (Telecommunication Computing Electronics and Control), vol. 14, n. 4, pp. 1217-1219, 2016.

[25] Nguyen, T. N., T. T. Duy, G.-T. Luu, P. T. Tran, and M. Voznak. "Energy Harvesting-based Spectrum Access with Incremental Cooperation, Relay Selection and Hardware Noises." Radioengineering 26, no. 1 (04, 2017): 240-50. doi:10.13164/re.2017.0240.

[26] Mckay, Matthew R., Alex J. Grant, and Iain B. Collings. "Performance Analysis of MIMO-MRC in DoubleCorrelated Rayleigh Environments." IEEE Transactions on Communications, 55, no. 3 (03 2007): 497-507. doi:10.1109/tcomm.2007.892450.

[27] Tran Hoang Quang Minh. "Hybrid Time-Power Switching Protocol of Energy Harvesting Bidirectional Relaying Network: Throughput and Ergodic Capacity Analysis." TELKOMNIKA (Telecommunication Computing Electronics and Control), 16, no. 5 (10, 2018): 189. http://dx.doi.org/10.12928/telkomnika.v16i5.9118.

[28] Table of Integrals, Series, and Products. (2015). doi:10.1016/c2010-0-64839-5. 